\title{
A candidate supermassive binary black hole system in the brightest cluster galaxy of RBS 797
}

\author{
M. Gitti ${ }^{1,2,3}$, M. Giroletti ${ }^{3}$, G. Giovannini ${ }^{1,3}$, L. Feretti ${ }^{3}$, and E. Liuzzo ${ }^{3}$ \\ 1 Physics and Astronomy Department, University of Bologna, via Ranzani 1, 40127 Bologna, Italy \\ e-mail: myriam.gitti@oabo.inaf.it \\ 2 INAF, Astronomical Observatory of Bologna, via Ranzani 1, 40127 Bologna, Italy \\ 3 INAF, Istituto di Radioastronomia di Bologna, via Gobetti 101, 40129 Bologna, Italy \\ Received 30 July 2013 / Accepted 14 August 2013
}

\section{ABSTRACT}

\begin{abstract}
Context. The radio source at the center of the cool-core galaxy cluster RBS $797(z=0.35)$ is known to exhibit a misalignment of its radio jets and lobes observed at different VLA scales, with the innermost $\sim$ kpc-scale jets being almost orthogonal to the radio emission which extends for tens of kpc filling the X-ray cavities. Gitti et al. (2006, A\&A, 448, 853) suggested that this peculiar radio morphology may indicate a recurrent activity of the central radio source, where the jet orientation is changing between the different outbursts due to the effects of supermassive binary black holes (SMBBHs).

Aims. We aim to reveal the nuclear radio properties of the brightest cluster galaxy (BCG) in RBS 797 and to investigate the presence of a SMBBH system in its center.

Methods. We performed new high-resolution observations at $5 \mathrm{GHz}$ with the European VLBI Network (EVN) on May 3, 2013, reaching an angular resolution of $\sim 9 \times 5 \mathrm{mas}^{2}$ and a sensitivity of $36 \mu \mathrm{Jy}$ beam ${ }^{-1}$. We also re-analyzed VLA archival data at $4.8 \mathrm{GHz}$ in A- and B-configurations.

Results. We report the EVN detection of two compact components in the BCG of RBS 797, with a projected separation of $\sim 77 \mathrm{pc}$. We can envisage two possible scenarios: the two components are two different nuclei in a close binary system, or they are the core and a knot of its jet. Both interpretations are consistent with the presence of SMBBHs. Our re-analysis of VLA archival data seems to favor the first scenario, as we detect two pairs of radio jets misaligned by $\sim 90$ degrees on the same $\sim \mathrm{kpc}$ scale emanating from the central radio core. If the two outbursts are almost contemporaneous, this is clear evidence of the presence of two active supermassive black holes whose radio nuclei are unresolved at VLA resolution. The nature of the double source detected by our EVN observations in the BCG of RBS 797 can be established only by future sensitive, multi-frequency VLBI observations. If confirmed, RBS 797 would be the first SMBBH system observed at medium-high redshift at VLBI resolution.
\end{abstract}

Key words. galaxies: active - galaxies: clusters: individual: RBS 797 - radio continuum: galaxies

\section{Introduction}

In the standard cold dark matter cosmological scenario, the formation of structures is a hierarchical process acting through galaxy mergers to form larger and larger structures, up to clusters of galaxies (e.g., White \& Rees 1978). During the merging of galaxies, the supermassive black holes (SMBHs) at their centers form binary systems, most of which, it is believed, eventually merge in less than a Hubble time (e.g., Komossa et al. 2003). An understanding of how SMBHs form and coalesce is important for the understanding of active galactic nuclei (AGN) dynamics as well as galaxy formation in general.

However, observational cases where both SMBHs in a merging system are accreting as AGNs are rare, and there have only been a few confirmed kpc-scale binary AGNs detected via various techniques (e.g., Komossa et al. 2003; Hudson et al. 2006; Koss et al. 2011; Fabbiano et al. 2011, where in the last case the separation is $\sim 150 \mathrm{pc}$ ). Recent simulations show that selection of double-peaked narrow emission lines is a promising method for identifying dual SMBH candidates, but high-resolution, multiwavelength follow-up observations are of critical importance for confirming the nature of the candidates (Blecha et al. 2013, and references therein). On the other hand, more compact binaries (with separations of less than few hundred pc) are extremely difficult to resolve with present telescopes, and only one object has been confirmed to host a pc-scale supermassive binary black hole (SMBBH) system (Rodriguez et al. 2006; Burke-Spolaor 2011).

The X-ray luminous galaxy cluster RBS $797(z=0.35)$ was discovered in the ROSAT All-Sky Survey (RASS, Schwope et al. 2000) and then observed by the optical ROSAT Bright Survey (RBS) during the identification of the X-ray RASS sources (Fischer et al. 1998; Schwope et al. 2000). Follow-up observations with Chandra revealed that it is a cool-core cluster, showing two symmetric X-ray minima (the so-called X-ray cavities) with diameters $\sim 20 \mathrm{kpc}$ and a bright $\mathrm{X}$-ray point-like source in the cluster center (Schindler et al. 2001). Comparison of the HST and Chandra images reveals that the central brightest cluster galaxy (BCG) that shows optical emission lines typical of AGNs (Fischer et al. 1998) coincides with the nuclear X-ray source. The optical BCG has the appearance of being bifurcated, perhaps because of an absorbing dust lane (Cavagnolo et al. 2011).

In the NRAO VLA Sky Survey (NVSS), an unresolved radio source of $20 \mathrm{mJy}$ is present at the center of RBS 797. The discovery of the X-ray cavity system has motivated in the past decade new radio observations at different frequencies and angular resolutions to study in detail the interaction of the central radio source with the intracluster medium (ICM). In particular, Gitti et al. (2006) performed VLA radio observations of RBS 797 at 1.4 and $4.8 \mathrm{GHz}$, ranging in resolution from a few tens of 
arcsec to subarcsec, reporting the detection of radio emission on different scales and orientations. The $1.4 \mathrm{GHz}$ radio emission imaged at $\sim 1$ arcsec resolution is found by these authors to fill the X-ray cavities nicely, extending along the northeastsouthwest direction up to $\sim 43 \mathrm{kpc}$ from the cluster center. This is the typical configuration observed in most cool-core clusters: the radio jets from the cluster central elliptical extend outwards in a bipolar flow, inflating lobes of radio-emitting plasma (radio bubbles) which push aside the X-ray emitting gas thus excavating depressions in the ICM which are detectable as apparent cavities in the X-ray images (see, e.g., Gitti et al. 2012; McNamara $\&$ Nulsen 2012, for recent reviews on AGN feedback in galaxy clusters). Remarkably, the innermost $4.8 \mathrm{GHz}$ radio jets imaged at subarcsec resolution are not aligned with the direction of the radio and X-ray emission seen at larger scale, but extend out from the core up to $\sim 13 \mathrm{kpc}$ and clearly show a north-south orientation, they are, therefore, almost perpendicular to the axis of the $1.4 \mathrm{GHz}$ emission filling the X-ray cavities (see Fig. 4 of Gitti et al. 2006). In-depth studies of the X-ray cavities and radio emission in RBS 797 have been presented by Gitti et al. (2006), Cavagnolo et al. (2011), and Doria et al. (2012). The cluster RBS 797 was also included in the cavity sample studies of Bîrzan et al. (2004, 2008), Rafferty et al. (2006), and Hlavacek-Larrondo et al. (2012). However, no definite explanation for the observed change in radio orientation has been presented so far.

We note that according to the existing radio images the central source does not show a slowly precessing jet, but a real change in the radio emission position angle (PA). Because this galaxy is the cluster BCG, we do not expect a large influence by galaxy motion. As already suggested by Gitti et al. (2006), we argue that this peculiar radio structure could be explained in the context of SMBBH models. The presence of a SMBBH system in a galactic nucleus may indeed become manifest through a change in the jet PA, which is likely originated by a spin flip of the primary SMBH caused perhaps by capture of a second SMBH, or to black hole mergers (Begelman et al. 1980; Merritt $\&$ Milosavljević 2005). Hence, relativistic material ejected in different episodes of activity may be expelled in different directions from the central engine, leaving a fossil record of the orientation history of the jets in the radio lobes. The radical change in the jet orientation may also be only apparent, being in fact due to the presence of two pairs of radio jets ejected in different directions by two close SMBHs, which are both active. In all these scenarios, the different radio directions observed in RBS 797 may thus be due to the presence of SMBBHs in the center of the BCG, unresolved at VLA resolution.

In this paper we present new high-resolution European Very Long Baseline Interferometry (VLBI) Network data of the radio source in the BCG of RBS 797 to study its nuclear properties and to investigate the presence of a SMBBH system in its center. We also show new Very Large Array (VLA) images at (sub-)arcsec resolutions produced by our re-analysis of combined archival data. With $\mathrm{H}_{0}=70 \mathrm{~km} \mathrm{~s}^{-1} \mathrm{Mpc}^{-1}$, and $\Omega_{\mathrm{M}}=1-\Omega_{\Lambda}=0.3$, the luminosity distance of RBS 797 is $1858 \mathrm{Mpc}$ and $1^{\prime \prime}$ corresponds to $\sim 4.8 \mathrm{kpc}$.

\section{Observations and data reduction}

We observed the radio core of the BCG in RBS 797 on May 3, 2013 (project code: RSG05, PI: M. Gitti) with a subset of the European VLBI Network (EVN) including the stations of Effelsberg, Medicina, Noto, Onsala, Shanghai, Torun, and Yebes. We observed at $5 \mathrm{GHz}$ using the e-VLBI technique,

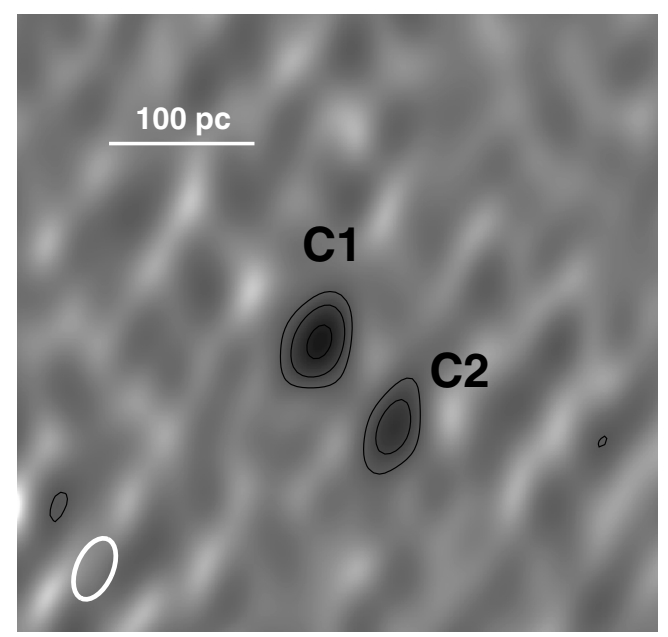

Fig. 1. $5 \mathrm{GHz}$ EVN map of the BCG in RBS 797 at a resolution of $9.4 \times 5.3 \mathrm{mas}^{2}$ in PA $-24^{\circ}$ (the beam is shown in the lower-left corner). The rms noise is $36 \mu \mathrm{Jy}_{\text {beam }}^{-1}$ and the peak flux density is $0.53 \mathrm{mJy}$ beam $^{-1}$. The contours levels start at $3 \sigma$ and increase by a factor of 2 . Two components, separated by 16 mas ( $\sim 77 \mathrm{pc})$, are clearly detected.

with data acquired and transmitted in real-time from the stations to the EVN data processor at JIVE. A bandwidth of 1 Gbps was sustained by most stations, corresponding to $128 \mathrm{MHz}$ divided into eight sub-bands, with two polarizations and 2-bit sampling.

We observed in phase reference mode, using the nearby $\left(d=1.85^{\circ}\right)$ source $\mathrm{J} 0954+7435$ as a phase calibrator, with $14 \times 4$-min scans on the target bracketed by $60 \mathrm{~s}$ scans on the calibrator, for a total net on source time of about $56 \mathrm{~min}$. We also observed four nearby NVSS sources (J094729+764355, $\mathrm{J} 093501+754526$, J094045+764833, and J095143+752952) to explore them as candidate phase reference sources for future observations; none of them turned out to host a compact component suitable for this purpose.

Correlation was performed in real time at JIVE; the JIVE pipeline was also used to carry out a priori amplitude calibration, automated flagging, and fringe fitting with Astronomical Image Processing System (AIPS) tasks. We edited the final visibility data and produced clean images in Difmap. Owing to the low flux density in the source, we did not attempt self-calibration.

The final beam size with natural weights is $9.4 \times 5.3 \mathrm{mas}^{2}$ in $\mathrm{PA}-24^{\circ}$. The image noise rms is $\sim 36 \mu \mathrm{Jy}_{\text {beam }}{ }^{-1}$, i.e., consistent with the expected thermal noise. Systematic amplitude calibration errors are typically at 5\%; therefore, we assume this uncertainty on the flux density measurements.

\section{Results}

Our 5 GHz EVN map of the BCG in RBS 797 is shown in Fig. 1. Two components are clearly detected. The first component, labeled $\mathrm{C} 1$, is located at RA: $09^{\mathrm{h}} 47^{\mathrm{m}} 12^{\mathrm{s}} .760$, Dec: $+76^{\circ} 23^{\prime} 13^{\prime \prime} .733$ and has a total flux density of $0.61 \mathrm{mJy}$. The second component, labeled $\mathrm{C} 2$, is separated by 16 mas $(76.8 \mathrm{pc})$ in PA $139^{\circ}$ and has a total flux density of $0.54 \mathrm{mJy}$. The results of the visibility model fit with two Gaussian components (Table 1) show that both components are compact and smaller than the observing beam. On the other hand, the size estimates provided by the model fit indicate that component $\mathrm{C} 2$ appears less compact than component $\mathrm{C} 1$ along the major axis, although it can still be considered unresolved given the size and shape of the beam.

With the aim of getting more insights into the global properties of the radio source, we also re-analyzed the archival 
Table 1. Gaussian model components.

\begin{tabular}{lccccccc}
\hline \hline Comp. & $\begin{array}{c}\text { Flux } \\
(\mathrm{mJy})\end{array}$ & $\begin{array}{c}r \\
(\mathrm{mas})\end{array}$ & $\begin{array}{c}\theta \\
(\mathrm{deg})\end{array}$ & $\begin{array}{c}a \\
(\mathrm{mas})\end{array}$ & $\begin{array}{c}a \\
(\mathrm{pc})\end{array}$ & $b / a$ & $\begin{array}{c}\Phi \\
(\mathrm{deg})\end{array}$ \\
\hline C1 & 0.606 & 0.43 & 29.4 & 2.96 & 14.2 & 1.00 & 58.1 \\
C2 & 0.540 & 16.1 & -139.2 & 7.44 & 35.7 & 0.59 & -1.4 \\
\hline
\end{tabular}

Notes. Column (1): Gaussian component as labeled in Fig. 1. Column (2): flux density. Columns (3), (4): polar coordinates of the center of the component relative to the observation pointing RA: $09^{\mathrm{h}} 47^{\mathrm{m}} 12^{\mathrm{s}} .760$, Dec: $+76^{\circ} 23^{\prime} 13^{\prime \prime}$. 740 . Columns (5), (6): major axis. Column (7): axial ratio. Column (8): component orientation. All angles are measured from north to east.

VLA A- and B-array data at $4.8 \mathrm{GHz}$. To fully exploit the relative advantages in terms of angular resolution and sensitivity of these two VLA configurations, we merged (with the AIPS task $\mathrm{DBCON}$ ) the two dataset to produce a new combined $\mathrm{A}+\mathrm{B}$ dataset at $4.8 \mathrm{GHz}$. We then produced images at different resolutions by specifying appropriate values of the parameters UVTAPER and ROBUST in the task IMAGR. Our new $4.8 \mathrm{GHz}$ VLA maps are shown in Fig. 2, superposed on the Chandra image of the central cluster region.

The subarcsec resolution image (black contours in Fig. 2, zoomed in the right panel) cleary shows the presence of another jet-like feature to the east, not detected in the previously published maps, which is the likely counterpart of the one to the west. In addition to the well-known nort-south jets, we therefore find strong evidence of the presence of $4.8 \mathrm{GHz}$ jets emanating also to the east-west direction. This east-west emission is best visible in the arcsec resolution image (green contours in Fig. 2); it extends up to $\sim 35 \mathrm{kpc}$ from the center and is clearly shown for the first time to nicely fill the X-ray cavity also at $4.8 \mathrm{GHz}$, besides the already known $1.4 \mathrm{GHz}$ emission (cf. Fig. 4 of Gitti et al. 2006).

\section{Discussion}

\subsection{Nature of the VLBI double source}

The detection of two compact components at VLBI resolution at the center of the BCG in RBS 797 is remarkable, especially when the short time of our observation is considered (only 56 min on source obtained over a limited hour angle range). By comparison, in a complete sample of 34 BCGs in nearby Abell clusters (distance class $<3$, Liuzzo et al. 2010) the 5 GHz VLBI detection rate is about $68 \%$, with only one case of double source. We note that this source, 3C 75 in A 400, is a dumbbell galaxy where the two components are separated by a much larger distance ( $\sim .2 \mathrm{kpc}$ in projection) than those detected in RBS 797.

The interpretation of the origin and nature of the double source in RBS 797 is complex, and with the current data we can envisage two different scenarios: (1) the two components $\mathrm{C} 1$ and $\mathrm{C} 2$ are two different nuclei in a close binary system, or (2) the two components are the core and a knot of its jet, and the emission from the underlying jet flow connecting the two components is not visible because of the limited sensitivity of our short observation. The presence of SMBBHs would be obvious in the first scenario, but given the VLA-scale properties of RBS 797 it would also be likely in the second scenario (see Sect. 4.2).

In the first scenario, we expect sensitive, multi-frequency VLBI observations to measure a flat spectrum for both components $\mathrm{C} 1$ and $\mathrm{C} 2$, and to reveal at least one jet emanating from one of them. If the two nuclear components are both active, each of them may show its own jet (or jet-counterjet pair) emerging
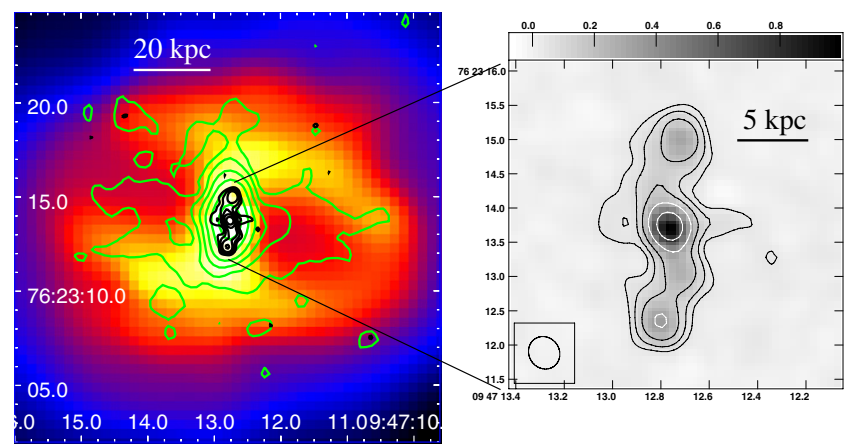

Fig. 2. The 4.8 GHz VLA contours obtained from the combined A- and B-array archival observations of RBS 797, imaged at different resolutions, are overlaid onto the Chandra image of the central region of the cluster (left panel). Green contours: $4.8 \mathrm{GHz}$ VLA map at 1'.38 × 1".33 resolution, obtained by setting ROBUST $=+5$, UVTAPER $=250$; the rms noise is $0.01 \mathrm{mJy}^{-1}$ beam $^{-1}$ and the contour levels are $0.03,0.06,0.12$, $0.24,0.48$, and $0.96 \mathrm{mJy}$ beam $^{-1}$; the total flux density is $\sim 4 \mathrm{mJy}$, with a peak flux density of $1.5 \mathrm{mJy}^{\text {beam }}{ }^{-1}$. Black contours (best visible in the zoom in the right panel): $4.8 \mathrm{GHz}$ VLA map at $0.49 \times 0.0^{\prime} 44$ resolution, obtained by setting ROBUST $=\mathbb{Q}$, UVTAPER $=\mathbb{Q}$; the rms noise is $0.01 \mathrm{mJy}$ beam $^{-1}$ and the contour levels are $0.04,0.08,0.16,0.32$, and $0.64 \mathrm{mJy}$ beam $^{-1}$; the total flux density is $\sim 2.8 \mathrm{mJy}$, with a peak flux density of $1.0 \mathrm{mJy}$ beam $^{-1}$.

from its center. We stress that only two cases, both at low redshift $(z<0.06)$, are known in the literature, namely 3C 75 in A 400 (Owen et al. 1985) and 0402+379 (Rodriguez et al. 2006), which is the only pc-scale SMBBH system discovered so far by the VLBI (Burke-Spolaor 2011). The detection of dual compact radio (or X-ray) sources in an active galaxy provides the most unambiguous evidence that a system hosts SMBBHs. Therefore, if the two compact radio components separated by only $\sim 77 \mathrm{pc}$ are confirmed to be two nuclei, RBS 797 would represent the first case of active SMBBHs observed at medium-high redshift at VLBI resolution. Considering that the sub-kpc-scale dual AGN may be relatively rare (as suggested by the recent observational work of Comerford et al. 2012), this detection would be particularly notable.

In the second scenario, component $\mathrm{C} 1$ is the most likely main core candidate because of its more compact size and the higher flux density (brightness temperature of $\sim 5 \times 10^{6} \mathrm{~K}$ ); we also estimate that $\mathrm{C} 2$ has a brightness temperature of $\sim 10^{6} \mathrm{~K}$, which is a reasonable value for a typical jet component. However, it is still possible that a knot is brighter than the nucleus itself, so the role of the nucleus-jet components in our interpretation may be inverted. A final test for this scenario can only be carried out by investigating the spectral properties of the two components, revealing a flat-spectrum core and a steep-spectrum jet knot. Deep observations may also reveal the presence of radio emission connecting the two features. However, we note that the orientation of the $\mathrm{C} 1-\mathrm{C} 2$ vector does not match any of the two PA of the large-scale radio emission (see Sect. 4.2).

With the current EVN data it is not possible to reach a definite conclusion about the origin and nature of the two compact components detected in the BCG of RBS 797, and their interpretation remains an open issue. However, from the morphology and orientation of the radio emission observed at VLA-scale we can gather elements in favor of one scenario rather than the other.

\subsection{Hints from archival VLA observations}

As first discovered by Gitti et al. (2006) and then confirmed by Bîrzan et al. (2008) and Cavagnolo et al. (2011), the central radio 
source in RBS 797 observed at VLA resolutions shows different orientations of the radio jets and lobes with scale. In particular, as seen in projection, the innermost $4.8 \mathrm{GHz}$ jets which extend to the north-south direction up to $\sim 13 \mathrm{kpc}$ from the radio core are almost orthogonal to the axis of the $1.4 \mathrm{GHz}$ radio emission filling the X-ray cavities and extending to the northeast-southwest direction up to $\sim 43 \mathrm{kpc}$ from the core. As a possible interpretation of this peculiar morphology, Gitti et al. (2006) suggested that the central radio source is experiencing recurrent activity, with misaligned jet axes from its different episodes, which could point to the effects of SMBBHs. These authors also noted the presence of a feature extending to the west out to a distance of $\sim 9 \mathrm{kpc}$ from the $4.8 \mathrm{GHz}$ core (see their Fig. 2c) that, if real, could be related to the $1.4 \mathrm{GHz}$ emission filling the cavities. We stress that, if confirmed, this western jet-like feature on $\sim \mathrm{kpc}$ scale would further strengthen the interpretation that RBS 797 harbors SMBBHs, as it may indicate the presence of ejecta originating from the secondary (active) SMBH.

Our re-analysis of the archival VLA A- and B-array data at $4.8 \mathrm{GHz}$ confirms that the western $4.8 \mathrm{GHz}$ jet structure is real and not an image artifact, indicating the presence of kpc-scale jets also emanating to the east-west direction (see black contours in Fig. 2). In the following discussion, we note that the $5 \mathrm{GHz}$ EVN map in Fig. 1 spans $\sim 80$ mas ( 400 pc), corresponding to a small region in the central part of the $5 \mathrm{GHz}$ VLA image in Fig. 2, which shows structures on scales of $\sim 20 \mathrm{kpc}$.

Since the large-scale east-west emission aligned with the $\mathrm{X}$-ray cavity axis appears to be more extended and diffuse than the north-south one (see green contours in Fig. 2), it should be older. This would be consistent with the fact that it has already created two large X-ray cavities, whereas the north-south emission is likely to be still in the process of excavating the ICM. In this context, Cavagnolo et al. (2011) report a hint of barely resolved structure in the Chandra image associated with the $4.8 \mathrm{GHz}$ radio structure, with residual $\mathrm{X}$-ray deficits just beyond the tips of the innermost radio jets, which might suggest potentially small cavities from a new outburst episode. This scenario would be consistent with the presence of a SMBBH system in which the observed radio emissions, elongated in different directions, are due to recurrent activity from a single central object that has changed ejection orientation because of the interaction with the secondary SMBH (due, for example, to merger or spin-flip, e.g., Merritt \& Milosavljević 2005, and references therein). The coalescent binaries able to alter the spin axis of the primary SMBH typically stall at a separation $(\sim 1 \mathrm{pc}$, Merritt \& Ekers 2002, and references therein) that is smaller than the observed separation ( $\sim 77 \mathrm{pc}$ ) of the two compact radio components in RBS 797. Therefore, in this picture the VLBI double source would probably be the core-jet structure of the primary SMBH (second scenario discussed in Sect. 4.1), whereas the secondary SMBH causing the spin-flip of the primary would remain undetected.

On the other hand, with the new combined $\mathrm{A}+\mathrm{B} 4.8 \mathrm{GHz}$ VLA images shown in Fig. 2 (black contours), we find a different orientation of the radio jets on the same $\sim k p c$ scale, indicating that the radio-emitting plasma on the east-west direction is still being freshly injected (in the absence of multi-frequency data needed for a detailed spectral aging analysis, we estimated a kinematic age of $\approx 10^{5}$ yrs by assuming a typical jet velocity of $\sim 0.1 \mathrm{c})$. The two outbursts could thus be almost contemporaneous, indicating the presence of two active SMBHs. This would support the first scenario discussed in Sect. 4.1. We also note that in the light of the VLA-scale properties, the core-jet hypothesis (the second scenario discussed in Sect. 4.1) is challenging since the pc-scale jet flow would not be aligned with any of the directions seen at kpc-scale in the VLA images.

\section{Conclusions}

Our main results can be summarized as follows:

- We report the VLBI detection of two compact radio components separated by $\sim 77 \mathrm{pc}$ in the BCG of the cool-core cluster RBS 797, obtained by our new EVN observations performed on May 3, 2013 at $5 \mathrm{GHz}$.

- Our re-analysis of the archival 4.8 GHz VLA data shows strong evidence of the presence of radio jets at subarcsec resolution also emanating to the east-west direction, in addition to the already known north-south jets. We therefore find different orientations of the jets on the same $\sim \mathrm{kpc}$ scale, indicating the likely presence of contemporaneous emission from two active SMBHs.

- We discuss the possible scenarios for the nature and origin of the VLBI double source, which suggest the presence of a SMBBH system. However, without detailed information on the morphological and spectral properties of the radio emission it is not possible to establish if the second component is in fact a different nucleus, or if it is a knot within the jet of the first nuclear component. In particular, in the first case we would expect to measure a flat spectrum for both components and to reveal jets emerging from each of them if they are both active.

New sensitive, high-resolution, multifrequency VLBI observations are essential to unveil the nuclear radio properties of the BCG in RBS 797, and represent the only way to confirm the presence of SMBBHs in its center.

Acknowledgements. We thank the referee for constructive comments that improved the presentation of the work. We acknowledge the financial contribution from contract ASI - I/009/10/0. The European VLBI Network (http://www . evlbi.org/) is a joint facility of European, Chinese, South African, and other radio astronomy institutes funded by their national research councils.

\section{References}

Begelman, M. C., Blandford, R. D., \& Rees, M. J. 1980, Nature, 287, 307 Bîrzan, L., Rafferty, D. A., McNamara, B. R., Wise, M. W., \& Nulsen, P. E. J. 2004, ApJ, 607, 800

Bîrzan, L., McNamara, B. R., Nulsen, P. E. J., Carilli, C. L., \& Wise, M. W. 2008, ApJ, 686, 859

Blecha, L., Loeb, A., \& Narayan, R. 2013, MNRAS, 429, 2594

Burke-Spolaor, S. 2011, MNRAS, 410, 2113

Cavagnolo, K. W., McNamara, B. R., Wise, M. W., et al. 2011, ApJ, 732, 71

Comerford, J. M., Gerke, B. F., Stern, D., et al. 2012, ApJ, 753, 42

Doria, A., Gitti, M., Ettori, S., et al. 2012, ApJ, 753, 47

Fabbiano, G., Wang, J., Elvis, M., \& Risaliti, G. 2011, Nature, 477, 431

Fischer, J., Hasinger, G., Schwope, A. D., et al. 1998, Astron. Nach., 319, 347

Gitti, M., Feretti, L., \& Schindler, S. 2006, A\&A, 448, 853

Gitti, M., Brighenti, F., \& McNamara, B. R. 2012, Adv. Astron., 2012

Hlavacek-Larrondo, J., Fabian, A. C., Edge, A. C., et al. 2012, MNRAS, 421, 1360

Hudson, D. S., Reiprich, T. H., Clarke, T. E., \& Sarazin, C. L. 2006, A\&A, 453, 433

Komossa, S., Burwitz, V., Hasinger, G., et al. 2003, ApJ, 582, L15

Koss, M., Mushotzky, R., Treister, E., et al. 2011, ApJ, 735, L42

Liuzzo, E., Giovannini, G., Giroletti, M., \& Taylor, G. B. 2010, A\&A, 516, A1

McNamara, B. R., \& Nulsen, P. E. J. 2012, New J. Phys., 14, 055023

Merritt, D., \& Ekers, R. D. 2002, Science, 297, 1310

Merritt, D. \& Milosavljević, M. 2005, Liv. Rev. Relativity, 8, 8

Owen, F. N., O'Dea, C. P., Inoue, M., \& Eilek, J. A. 1985, ApJ, 294, L85

Rafferty, D. A., McNamara, B. R., Nulsen, P. E. J., \& Wise, M. W. 2006, ApJ, 652,216

Rodriguez, C., Taylor, G. B., Zavala, R. T., et al. 2006, ApJ, 646, 49

Schwope, A., Hasinger, G., Lehmann, I., et al. 2000, Astron. Nachr., 321, 1

White, S. D. M., \& Rees, M. J. 1978, MNRAS, 183, 341 